\title{
Drug Interaction Potential of Osilodrostat (LCI699) Based on Its Effect on the Pharmacokinetics of Probe Drugs of Cytochrome P450 Enzymes in Healthy Adults
}

\author{
Sara Armani ${ }^{1}$ - Lillian Ting ${ }^{2,5}$ - Nicholas Sauter ${ }^{2}$ Christelle Darstein ${ }^{3}$. \\ Anadya Prakash Tripathi ${ }^{4,6} \cdot$ Lai Wang ${ }^{2} \cdot$ Bing $\mathrm{Zhu}^{2} \cdot$ Helen $\mathrm{Gu}^{2}$. \\ Dung Yu Chun ${ }^{2} \cdot$ Heidi J Einolf ${ }^{2}$ - Swarupa Kulkarni ${ }^{2}$
}

Published online: 2 February 2017

(C) The Author(s) 2017. This article is published with open access at Springerlink.com

\begin{abstract}
Background and Objectives Osilodrostat (LCI699) is an adrenal steroidogenesis inhibitor currently in late-phase clinical development as a potential treatment for Cushing's disease. This study evaluated the inhibitory effect of osilodrostat on the pharmacokinetics of probe substrates of the cytochrome P450 (CYP) enzymes CYP1A2, CYP2C19, CYP2D6, and CYP3A4.

Methods Healthy adult volunteers received single-dose cocktail probe substrates [caffeine $(100 \mathrm{mg})$, omeprazole $(20 \mathrm{mg})$, dextromethorphan $(30 \mathrm{mg})$, and midazolam $(2 \mathrm{mg})$ ] followed by a 6-day washout. Subjects then received a single dose of osilodrostat $50 \mathrm{mg}$ followed by a single dose of cocktail probe substrates.

Results Nineteen of twenty subjects (ten were male) completed the study. Mean age, body weight, and body mass index were 41.8 years, $73.0 \mathrm{~kg}$, and $24.4 \mathrm{~kg} / \mathrm{m}^{2}$. Geometric mean ratio of the area under the concentration-time curve
\end{abstract}

Electronic supplementary material The online version of this article (doi:10.1007/s40261-017-0497-0) contains supplementary material, which is available to authorized users.

Swarupa Kulkarni

swarupa.kulkarni@novartis.com

PAREXEL International GmbH, Berlin, Germany

2 Novartis Pharmaceuticals Corporation, 1 Health Plaza, Building 315, 04-4230E, East Hanover, NJ 07936, USA

3 Novartis Pharma AG, Basel, Switzerland

4 Novartis Healthcare Pvt. Ltd, Hyderabad, India

5 Present Address: Currently at Merck \& Co., Rahway, NJ, USA

6 Present Address: Currently at PAREXEL International Pvt. Ltd, Hyderabad, India from time zero to the last measureable concentration and $90 \%$ confidence intervals of probe substrate exposure with osilodrostat were: caffeine (CYP1A2 probe substrate), 2.33 (2.10-2.59); omeprazole (CYP2C19), 1.91 (1.74-2.11); dextromethorphan (CYP2D6), 1.48 (1.34-1.63); and midazolam (CYP3A4/5), 1.50 (1.41-1.60). Corresponding values for geometric mean ratio of maximum plasma concentration (90\% confidence interval) for the change in substrate exposure were $1.07(0.988-1.15), 1.61$ (1.40-1.84), 1.35 (1.21-1.50), and 1.47 (1.32-1.62).

Conclusions Osilodrostat is a moderate inhibitor of CYP1A2 and CYP2C19 and a weak inhibitor of CYP2D6 and the most clinically important CYP enzyme, CYP3A4. Osilodrostat is unlikely to significantly increase the exposures of other medications cleared by CYP3A4. These findings are clinically relevant given that Cushing's disease is a chronic condition often requiring multiple medications and that most other therapies have significant drug interaction potential.

\section{Key Points}

Osilodrostat (LCI699) is currently in late-phase clinical development as a potential treatment for Cushing's disease. In this study, osilodrostat was shown to be a weak-to-moderate inhibitor of four important human cytochrome P450 (CYP) enzymes: CYP1A2, CYP2C19, CYP2D6, and CYP3A4/5.

Given that patients with Cushing's disease often require multiple medications to treat diseaseassociated co-morbidities, these findings are clinically relevant and further support osilodrostat as a potential new treatment for this disorder. 


\section{Introduction}

Cushing's disease is a rare endocrine disorder caused by an adrenocorticotropic hormone-secreting pituitary tumor. As adrenocorticotropic hormone controls the release of cortisol from the adrenal glands, excess adrenocorticotropic hormone secreted by the tumor increases cortisol production, resulting in a hypercortisolemic state. Treatment for Cushing's disease aims to normalize cortisol levels and reverse the signs and symptoms of hypercortisolism [1]. The primary treatment is transsphenoidal pituitary surgery; medical therapy or pituitary irradiation is used when there is persistent or recurrent hypercortisolism after surgery [1]. Although a number of medical therapies are available to treat Cushing's disease [2-4], choosing an appropriate agent for the individual patient requires numerous considerations. As patients with Cushing's disease typically experience a high burden of illness, many are likely to be taking multiple medications such as statins and anti-hypertensive medications, thus there is an increased risk of drug-drug interactions (DDIs). DDIs can result in profound clinical effects, either by decreasing drug efficacy or by augmenting toxicity. Thus, one key consideration in choosing a medical therapy for treating hypercortisolism in Cushing's disease is an awareness of the potential for DDIs with concomitant medications.

Osilodrostat (LCI699) is an oral inhibitor of 11ß-hydroxylase [cytochrome P450 (CYP) 11B1], the enzyme that catalyzes the final step in cortisol synthesis in the adrenal cortex [5]. Osilodrostat also blocks aldosterone synthesis via inhibition of aldosterone synthase (CYP11B2) [6, 7]. Osilodrostat has a half-life of 3-5 h [5], allowing for twice-daily dosing, and has been shown to effectively decrease urinary free cortisol (UFC) levels in patients with Cushing's disease [8, 9]. In a 10-week, proofof-concept study (LINC 1), osilodrostat (2-30 mg twice daily, titrated according to patient response) normalized UFC in 92\% (11/12) of patients with Cushing's disease [8]. Reductions in UFC were confirmed over a longer follow-up period in the LINC 2 study: osilodrosat normalized UFC in $79 \%(15 / 19)$ of patients after 22 weeks of treatment [9]. The effect of osilodrostat in larger patient populations is currently under evaluation in the confirmatory phase III studies LINC 3 and LINC 4.

As previous in vitro studies have suggested that osilodrostat has inhibitory potential against certain CYP enzymes (Supplemental Information), a pharmacokinetic study was conducted in healthy adult subjects to characterize the clinical drug interaction potential of osilodrostat for CYP1A2, CYP2C19, CYP2D6, and CYP3A4/5 with the respective probe substrates caffeine, omeprazole, dextromethorphan, and midazolam.

\section{Methods}

\subsection{Study Population and Design}

This was a single-center, phase I, open-label, fixed-sequence, DDI study in healthy adult volunteers: male and female individuals aged between 18 and 55 years with systolic and diastolic blood pressure, pulse rate, and body temperature within normal ranges, body weight between 50 and $100 \mathrm{~kg}$, and body mass index between 18 and $33 \mathrm{~kg} / \mathrm{m}^{2}$ were recruited. Subjects were excluded from the study if they had used or consumed substances known to interfere with those CYP enzymes being investigated.

The study consisted of two treatment periods. In period 1 (day 1), each subject received a single dose of the probe drug cocktail (modified Cooperstown cocktail) orally, which contained caffeine $(100 \mathrm{mg})$, omeprazole $(20 \mathrm{mg})$, dextromethorphan $(30 \mathrm{mg})$, and midazolam $(2 \mathrm{mg})$. After a washout period of 6 days, in period 2 (day 8), subjects received a single 50-mg dose of osilodrostat orally followed by a single dose of probe drug cocktail 30 min later. Blood samples were collected after administration of the cocktail in both study periods at the following time points: 0 (pre-dose), $0.25,0.5,1,1.5,2,2.5,3,4,6,8,12,24$, and $48 \mathrm{~h}$ post-dose. Plasma concentrations of the cocktail probe substrates were assayed using validated methods (WuXi AppTec Co., Ltd). The analytes were extracted from plasma samples and analysis was performed by validated liquid chromatography-tandem mass spectrometry assay. The limits of quantitation for the different analytes were $25.0 \mathrm{ng} / \mathrm{mL}$ for caffeine, $0.1 \mathrm{ng} / \mathrm{mL}$ for dextromethorphan, $3.0 \mathrm{ng} / \mathrm{mL}$ for omeprazole, $0.05 \mathrm{ng} / \mathrm{mL}$ for midazolam, and $1.0 \mathrm{ng} / \mathrm{mL}$ for osilodrostat. Plasma concentrations of osilodrostat were assayed using a validated liquid chromatography-tandem mass spectrometry assay, with a limit of quantitation of $1.00 \mathrm{ng} / \mathrm{mL}$ [10].

\subsection{Analysis Sets}

The safety set included all subjects who received at least one dose of study medication. Five separate pharmacokinetic analysis sets were evaluated, one for each probe drug substrate and one for osilodrostat. For each probe substrate, the pharmacokinetic analysis set included all subjects who received the planned amount of probe substrate in period 1 or the planned amount of probe substrate plus osilodrostat in period 2, did not vomit within $4 \mathrm{~h}$ of study drug administration, and had sufficient pharmacokinetic concentration data to determine at least one evaluable primary pharmacokinetic parameter. The pharmacokinetic analysis set for osilodrostat included all subjects who received the planned amount of osilodrostat in period 2, did not vomit 
within $4 \mathrm{~h}$ of study drug administration, and had at least one evaluable pharmacokinetic parameter.

\subsection{Pharmacokinetic Assessment}

The primary objective was to assess the effect of osilodrostat on the pharmacokinetic parameters of the CYP1A2, CYP2C19, CYP2D6, and CYP3A4/5 probe substrates caffeine, omeprazole, dextromethorphan, and midazolam using a modified Cooperstown cocktail in healthy adult subjects. Secondary objectives included: determination of key pharmacokinetic parameters [area under the concentration-time curve (AUC) from time zero to the last measureable concentration $\left(\mathrm{AUC}_{\text {last }}\right), \mathrm{AUC}$ from time zero extrapolated to infinity $\left(\mathrm{AUC}_{\mathrm{inf}}\right)$, and maximum plasma concentration $\left(C_{\max }\right)$ ] for metabolites of the probe substrates [i.e., paraxanthine (caffeine), dextrorphan (dextromethorphan), 5-hydroxyomeprazole (omeprazole), and hydroxymidazolam (midazolam)]; pharmacokinetic exposure of a single 50-mg dose of osilodrostat; and safety and tolerability of osilodrostat when co-administered with probe substrates. All pharmacokinetic parameters of osilodrostat and cocktail probe substrates were determined by non-compartmental analysis using Phoenix WinNonlin version 6.2 .

\subsection{Safety and Tolerability}

Safety assessment was based mainly on the frequency of adverse events (AEs), as reported by the investigator at any time from informed consent until 30 days after the last dose of osilodrostat was administered, and on the number of laboratory values that fell outside of pre-determined ranges. Laboratory tests were performed at screening and at the end-of-treatment visit (study day 10). All AEs were coded according to Common Terminology Criteria for Adverse Events version 4.03. Treatment-emergent AEs were defined as those that started on or after study drug administration but not after 30 days from the date of study drug administration, or those that started before study drug administration and worsened afterwards.

\subsection{Statistical Analyses}

Statistical analyses of the pharmacokinetic parameters for the primary objective were performed using the pharmacokinetic analysis set for each probe drug. The single-dose pharmacokinetics of each of the probe drugs administered with and without a single 50-mg dose of osilodrostat was compared using a model-based statistical analysis. The single-dose pharmacokinetic parameters $\mathrm{AUC}_{\text {last }}, \mathrm{AUC}_{\mathrm{inf}}$, and $C_{\max }$ for the CYP1A2, CYP2C19, CYP2D6, and CYP3A4/5 substrates (caffeine, dextromethorphan, omeprazole, and midazolam) were log transformed and analyzed separately for each probe drug with a linear mixed-effects model. For the DDI analysis, the point estimate for the treatment difference in least-squares means (for the log pharmacokinetic parameters) and the corresponding $90 \%$ confidence intervals (CIs) were calculated to obtain the point estimate and $90 \%$ CI for the ratio of geometric means (for the pharmacokinetic parameters) of the test compared with the reference [(probe plus osilodrostat $50 \mathrm{mg}$ )/probe alone]. The $90 \%$ CIs for the ratio of geometric means for $\mathrm{AUC}_{\text {last }}, \mathrm{AUC}_{\mathrm{inf}}$, and $C_{\max }$ of substrates with and without osilodrostat co-administration were determined. Descriptive statistics were reported for all secondary pharmacokinetic analyses.

\subsection{Physiologically Based Pharmacokinetic Modeling of Osilodrostat Pharmacokinetics and Drug-Drug Interactions}

A physiologically based pharmacokinetic (PBPK) model was developed for osilodrostat within the framework of the Simcyp ${ }^{\circledR}$ Simulator (version 15, release 1; Certara Inc.). The model incorporated physiochemical and clinical pharmacokinetic parameters, in vitro and in vivo enzyme phenotyping information, and in vitro CYP perpetrator (inhibition and induction) characteristics of osilodrostat. The Supplemental Information contains details of the methods and results from the in vitro CYP inhibition (Supplemental Table 1), CYP induction (Supplemental Tables 2 and 3), CYP enzyme reaction phenotyping studies (Supplemental Table 4), and the input parameters for the PBPK model (Supplemental Table 5). The model was developed to simulate the concentration-time profiles and pharmacokinetic parameters of osilodrostat after 30- and 50-mg single doses. In addition, the model was developed to simulate the pharmacokinetic parameters and the geometric mean AUC and $C_{\max }$ ratios of the CYP probe substrates when co-administered with a single 50-mg dose of osilodrostat according to the actual clinical trial design described above. The qualified model was then used to predict the pharmacokinetics of osilodrostat after multiple 30-mg twice-daily doses (14 days), and to predict the DDIs of the CYP probe substrates dosed on day 14 after multiple doses of osilodrostat (30 mg twice daily, dosed on days $1-16)$.

\section{Results}

\subsection{Patient Population}

Twenty subjects entered the study, of whom 19 completed it (one subject discontinued the study because of 
withdrawal of consent). The median age was 41.5 years (range 27-55 years); most subjects (95\%) were Caucasian, ten subjects were male, and the mean (standard deviation) body mass index and weight were $24.4(2.9) \mathrm{kg} / \mathrm{m}^{2}$ and $73.0(12.9) \mathrm{kg}$, respectively.

\subsection{Effect of Osilodrostat on the Pharmacokinetics of Probe Cytochrome P450 Substrates}

Co-administration of each probe substrate with a single 50-mg dose of osilodrostat resulted in a modest increase in exposure vs. probe substrate alone (Fig. 1). When caffeine was co-administered with osilodrostat, higher caffeine concentrations were observed vs. caffeine alone (Fig. 2a). Although large variations were observed at each time point for both omeprazole and dextromethorphan when co-administered with osilodrostat, there was an overall increase in omeprazole and dextromethorphan exposures (Fig. 2b, c, respectively). Midazolam co-administered with osilodrostat produced a slight increase in midazolam exposure compared with midazolam alone (Fig. 2d). The increased exposure of caffeine, omeprazole, dextromethorphan, and midazolam following co-administration with osilodrostat indicated a modest inhibitory effect of osilodrostat on each probe substrate (Table 1).

\subsection{Secondary Pharmacokinetic Results}

Caffeine, omeprazole, and midazolam were eliminated more slowly when co-administered with osilodrostat vs. alone, as indicated by a longer half-life and a 61,30 , and $33 \%$ decrease in apparent total clearance of the drug from plasma after oral administration, respectively (Table 1).
Dextromethorphan data for these parameters were affected by large inter-patient variations (data not shown), mainly owing to two outliers with extreme values, consistent with subjects who are poor metabolizers of dextromethorphan.

There was a reduction in metabolite formation of all four probe substrates following co-administration of osilodrostat compared with administration of the probe substrates alone. Caffeine, omeprazole, dextromethorphan, and midazolam metabolite ratios were reduced by 62,21 , 35 , and $14 \%$, respectively.

\subsection{Pharmacokinetic Exposure of Osilodrostat}

Following a single oral dose of osilodrostat $50 \mathrm{mg}$, peak plasma concentration occurred at $\sim 1.53 \mathrm{~h}$. The geometric mean (coefficient of variation) of $\mathrm{AUC}_{\mathrm{inf}}$ and $C_{\max }$ was $3430 \mathrm{ng} \cdot \mathrm{h} / \mathrm{mL}(30.2 \%)$ and $392 \mathrm{ng} / \mathrm{mL}(21.4 \%)$, respectively, with a half-life of $4.73 \mathrm{~h}$.

\subsection{Safety and Tolerability}

All 20 subjects were included in the safety analysis set. Of these, at least one $\mathrm{AE}$ was experienced by eight subjects $(40 \%)$. AEs suspected to be drug related were reported by six subjects (30\%); fatigue (three subjects; $15 \%$ ) and dizziness (two subjects; $10 \%$ ) were most common, with one subject reporting flatulence and diarrhea $(5 \%)$. These AEs resolved on the day of onset (except flatulence, which resolved on the next day after onset) without any action. All but one of the suspected AEs were of grade 1 severity; one AE of dizziness was of grade 2 severity. No grade 3 or 4 AEs, or serious AEs, were reported.

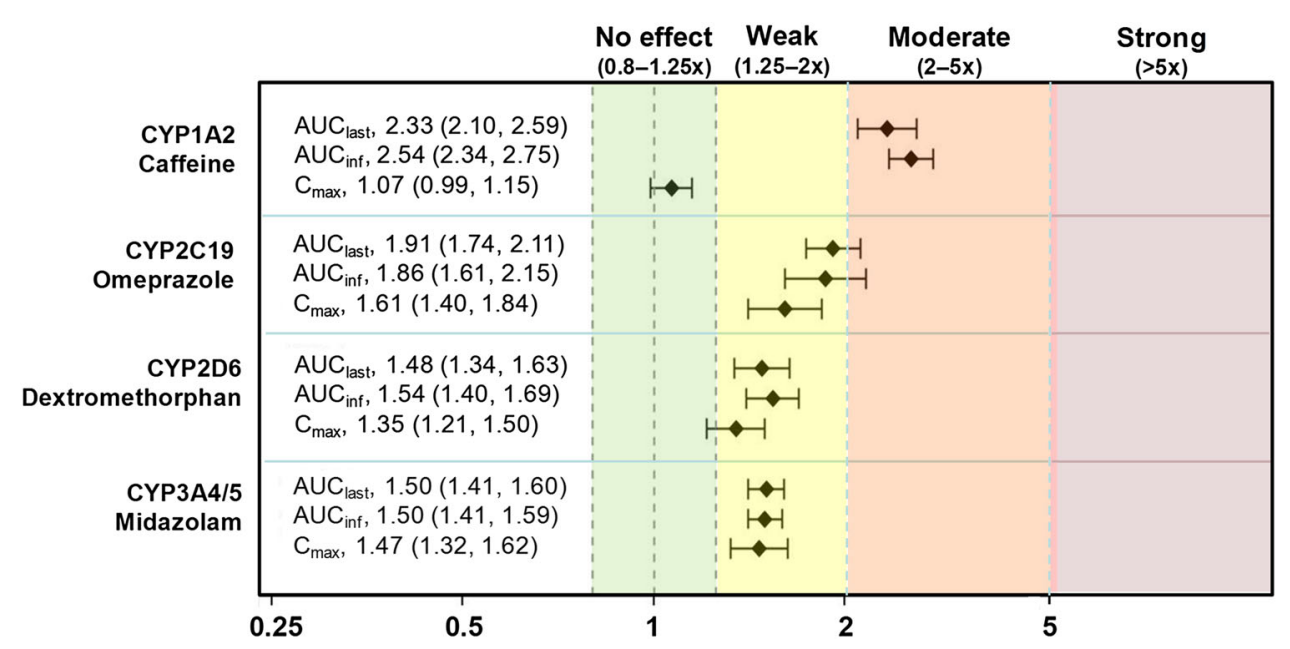

Fig. 1 Inhibitory effect of a single 50-mg dose of osilodrostat on cytochrome P450 (CYP) probe substrates based on definitions provided by US Food and Drug Administration guidelines [22]. Geometric mean ratios (90\% confidence interval) of (substrate plus osilodrostat)/substrate alone for each pharmacokinetic parameter are shown. $A U C_{i n f}$ area under the concentration-time curve from time zero extrapolated to infinity, $A U C_{\text {last }}$ area under the concentrationtime curve from time zero to the last measureable concentration, $C_{\max }$ maximum plasma concentration, $C Y P$ cytochrome $\mathrm{P} 450$ 

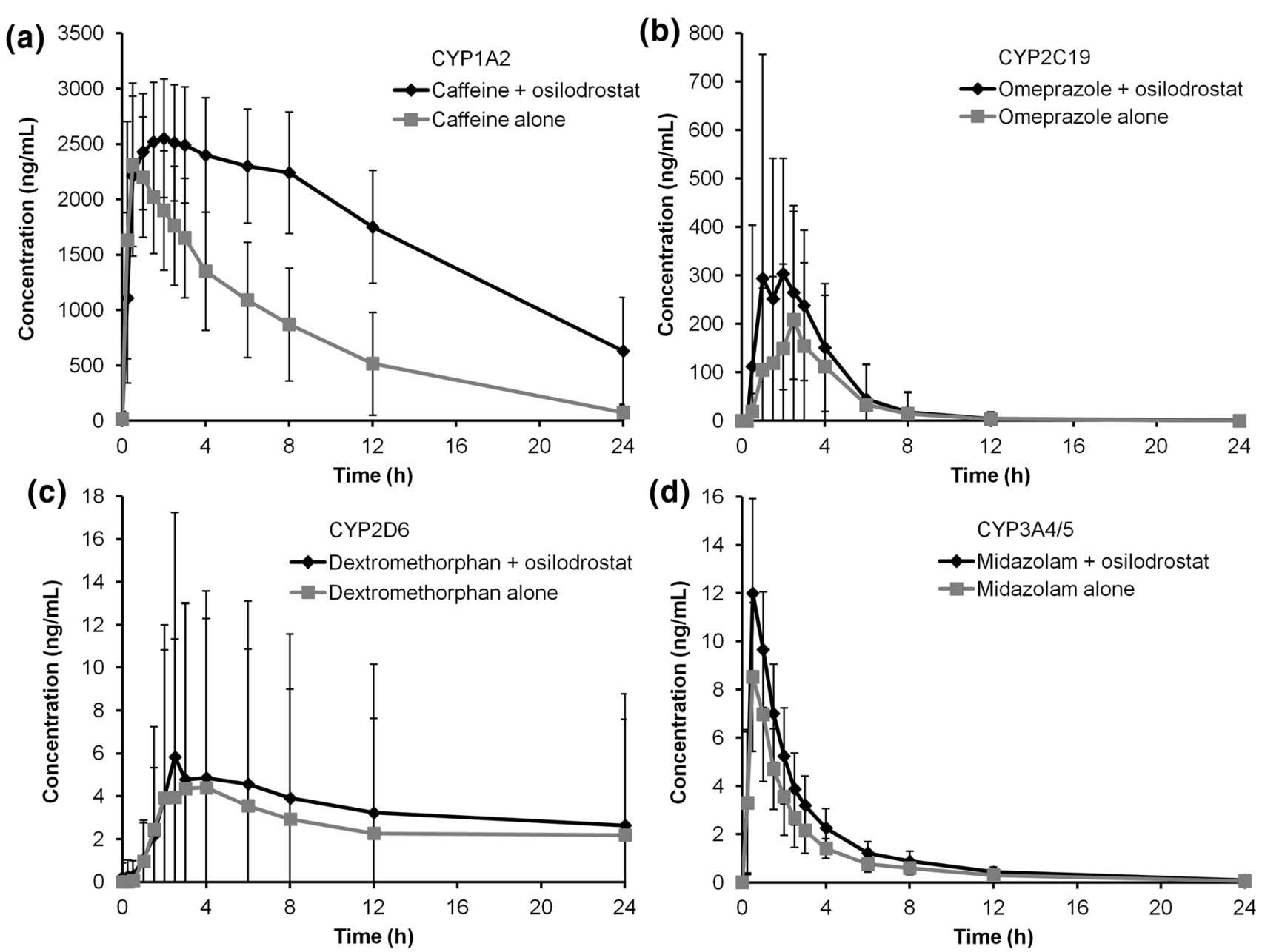

Fig. 2 Arithmetic mean concentration-time profiles of a caffeine, b omeprazole, $\mathbf{c}$ dextromethorphan, and $\mathbf{d}$ midazolam given alone vs. with osilodrostat. Error bars represent standard deviation. Samples

\subsection{Physiologically Based Pharmacokinetic Modeling of Osilodrostat Pharmacokinetics and Drug-Drug Interactions}

The PBPK model was qualified to simulate the concentration-time profiles and pharmacokinetic parameters of osilodrostat 30- and 50-mg single oral doses (Supplemental Fig. 1 and Supplemental Table 6). The PBPK-modeled pharmacokinetic parameters were within $15 \%$ of the observed values. This model was further qualified to simulate the DDI of a single 50-mg osilodrostat dose on the individual CYP probe substrates according to the actual trial design. The model simulated the geometric mean AUC ratios within $30 \%$ of the observed value for all CYP substrates (Table 2). With this qualified model, osilodrostat (30 mg twice daily) concentration-time profile and pharmacokinetic parameters were predicted up to 14 days of dosing (Supplemental Fig. 2 and Supplemental Table 6). After multiple 30-mg twice-daily doses of osilodrostat for pharmacokinetic analysis were collected up to $48 \mathrm{~h}$; concentration-time profiles are shown up to $24 \mathrm{~h}$. CYP cytochrome $\mathrm{P} 450$

(days 1-16) with a single dose of CYP substrates on day 14 , the model predicted a similar DDI effect to that with a single 50-mg dose of osilodrostat for the CYP1A2, CYP2D6, and CYP3A4/5 substrates $(\leq 3 \%$ change; Table 2). For CYP2C19, a modest increase of $26 \%$ in the geometric mean AUC ratio was predicted after $30 \mathrm{mg}$ twice-daily multiple osilodrostat doses compared with a single 50-mg dose. This predicted increase after multiple osilodrostat doses was a result of the additional time-dependent nature of CYP2C19 inhibition by osilodrostat.

\section{Discussion}

CYP enzymes are responsible for metabolizing most therapeutic agents [11]. Although 58 human CYP genes have been identified to date [11], only a minority are involved in most therapeutic drug metabolism [12]. Of the 200 most frequently prescribed medications in the USA, 37\% are 
Table 1 Summary of primary and secondary pharmacokinetic parameters for probe substrates with and without a single 50-mg dose of osilodrostat (pharmacokinetic analysis set)

\begin{tabular}{|c|c|c|c|c|c|c|}
\hline Probe substrate & Treatment & $\mathrm{AUC}_{\text {last }}(\mathrm{ng} \cdot \mathrm{h} / \mathrm{mL})$ & $\mathrm{AUC}_{\mathrm{inf}}(\mathrm{ng} \cdot \mathrm{h} / \mathrm{mL})$ & $C_{\max }(\mathrm{ng} / \mathrm{mL})$ & Half-life (h) & $\mathrm{CL} / \mathrm{F}(\mathrm{L} / \mathrm{h})$ \\
\hline \multirow[t]{2}{*}{ Caffeine (CYP1A2) } & Substrate alone & $17,200(61.7)$ & $16,200(44.8)$ & $2500(29.5)$ & $5.39(58.1)$ & $6.18(44.8)$ \\
\hline & Substrate plus osilodrostat & $41,100(37.5)$ & $41,900(32.1)$ & $2640(20.1)$ & $7.39(48.4)$ & $2.39(32.1)$ \\
\hline \multirow[t]{2}{*}{ Omeprazole (CYP2C19) } & Substrate alone & $460(97.0)$ & 618 (92.9) & $271(69.7)$ & $0.77(48.4)$ & $32.3(92.9)$ \\
\hline & Substrate plus osilodrostat & $852(90.7)$ & 885 (89.9) & $420(70.8)$ & $0.87(43.9)$ & $22.6(89.9)$ \\
\hline \multirow{2}{*}{$\begin{array}{l}\text { Dextromethorphan } \\
\text { (CYP2D6) }\end{array}$} & Substrate alone & $7.63(1758.1)$ & $22.8(193.8)$ & $1.24(330.9)$ & $7.63(87.5)$ & $1310(193.8)$ \\
\hline & Substrate plus osilodrostat & $12.6(1857.4)$ & $20.0(233.9)$ & $1.80(332.6)$ & $7.71(107.4)$ & $1500(233.9)$ \\
\hline \multirow[t]{2}{*}{ Midazolam (CYP3A4/5) } & Substrate alone & $21.2(40.2)$ & $22.0(39.6)$ & $8.78(38.8)$ & $4.44(38.3)$ & $91.0(39.6)$ \\
\hline & Substrate plus osilodrostat & $31.7(34.2)$ & $32.8(34.1)$ & $12.8(26.9)$ & $4.81(35.1)$ & $61.0(34.1)$ \\
\hline
\end{tabular}

Data are shown as geometric mean $(\mathrm{CV} \%)$

$A U C_{i n f}$ area under the concentration-time curve from time zero extrapolated to infinity, $A U C_{\text {last }}$ area under the concentration-time curve from time zero to the last measureable concentration, $C L / F$ apparent total clearance, $C_{\max }$ maximum plasma concentration, $C V \%$ coefficient of variation, CYP cytochrome $\mathrm{P} 450$

Table 2 Physiologically based pharmacokinetic model-predicted effects of osilodrostat on cytochrome P450 (CYP) substrates after single (50$\mathrm{mg}$ ) or multiple (30-mg twice-daily) doses of osilodrostat

\begin{tabular}{llll}
\hline Probe substrate & Treatment $^{\mathrm{a}}$ & \multicolumn{2}{l}{ Predicted geometric mean ratio (prediction error, \%) } \\
\cline { 2 - 3 } & & AUC $_{\text {last }(48 \mathrm{~h})}$ & $C_{\text {max }}$ \\
\hline Caffeine (CYP1A2) & Single dose (osilodrostat 50 mg) & $1.66(-29 \%)$ & $1.17(+9.3 \%)$ \\
& Multiple dose (osilodrostat 30 mg twice daily) & 1.67 & 1.15 \\
Omeprazole (CYP2C19) & Single dose (osilodrostat 50 mg) & $1.55(-19 \%)$ & $1.44(-11 \%)$ \\
& Multiple dose (osilodrostat 30 mg twice daily) & 1.95 & 1.73 \\
Dextromethorphan (CYP2D6) & Single dose (osilodrostat 50 mg) & $1.47(-0.7 \%)$ & $1.43(+5.9 \%)$ \\
& Multiple dose (osilodrostat 30 mg twice daily) & 1.52 & 1.47 \\
Midazolam (CYP3A4/5) & Single dose (osilodrostat 50 mg) & $1.49(-0.67 \%)$ & $1.39(-5.4 \%)$ \\
& Multiple dose (osilodrostat 30 mg twice daily) & 1.51 & 1.45 \\
\hline
\end{tabular}

$A U C_{\text {last }(48 \mathrm{~h})}$ area under the concentration-time curve from time zero to the last measureable concentration at $48 \mathrm{~h}, C_{\max }$ maximum plasma concentration

${ }^{\text {a }}$ Simulated single dose of osilodrostat $50 \mathrm{mg}$ plus substrate on day 1 or predicted multiple doses of osilodrostat $30 \mathrm{mg}$ twice daily on days $1-16$ plus a single dose of substrate on day 14

${ }^{\mathrm{b}}$ Calculated prediction error $(\%)=[$ (predicted value - observed value $) /$ observed value $] \times 100$; observed values are shown in Fig. 2

metabolized by CYP3A, followed by CYP2C9 (17\%), CYP2D6 (15\%), CYP2C19 (10\%), and CYP1A2 (9\%), with CYP2C8, CYP2B6, and other CYP isoforms metabolizing the remainder [12]. The CYP3A family is arguably the most clinically significant, not only because of their majority role in drug metabolism, but also because they are the most abundant CYP enzymes in human liver, comprising 30-50\% of total CYP content [13]. Four CYP3A enzymes exist in humans: CYP3A4, CYP3A5, CYP3A7, and CYP3A43 [14], with CYP3A4/5 being the most predominant isoforms in adult liver [15].

Inhibition or induction of CYP enzymes may result in clinically significant DDIs, leading to toxicity or therapeutic failure. These potentially serious consequences have prompted regulatory agencies to issue guidance on characterizing the DDI potential of new molecular entities
[16, 17]. In vitro screening for investigative compounds that inhibit CYP enzymes is a well-known methodology for predicting drug interaction potential in vivo. Based on previous in vitro assessments, osilodrostat has inhibitory potential against CYP1A2, CYP2C19, CYP2D6, and CYP3A4/5. The current study was therefore conducted in human subjects to further characterize the potential inhibitory effects of osilodrostat on these key human CYP enzymes.

Although osilodrostat is in late-phase clinical development as a treatment for Cushing's disease, the rarity of the disorder was expected to affect the study recruitment rate, thus healthy volunteers were enrolled in the DDI study instead. Additionally, the doses of osilodrostat in patients with Cushing's disease are not fixed; they are titrated for each subject according to their response up to a maximum 
of $30 \mathrm{mg}$ twice daily. In addition, the dose requirement in an individual subject may change over time. It is for this reason that a cross-over DDI study with a fixed dose of osilodrostat would not have been feasible in the Cushing's disease population.

Regulatory agencies recommend a cocktail approach to screening for potential in vivo DDIs. Simultaneous administration of multiple in vivo probes of drug-metabolizing enzymes offers several distinct advantages such as time and cost savings, minimizing the confounding influence of inter- and intra-individual variability over time. Substrates for the CYP enzymes were chosen based on considerations that the substrates are specific to individual CYP enzymes, and that simultaneous administration of these multiple substrates does not result in mutual drug interactions [18].

As osilodrostat is a potent inhibitor of cortisol synthesis, multiple dosing without intervention was not feasible, thus a single-dose design that provided adequate coverage of steady-state exposure at the current highest clinical dose (30 mg twice daily) was used. Through modeling and simulation of single-dose osilodrostat pharmacokinetics and DDI on the CYP probe substrates, the effect of multiple-dose DDI of osilodrostat was predicted. PBPK modeling offers the ability to bridge clinically tested situations to those that are more challenging or impossible to conduct [19-21]. Based on the in vitro data and extrapolation in vivo, time-dependent inhibition/induction effects of LCI699 are weak; therefore, similar DDI was predicted after single $(50 \mathrm{mg})$ and multiple $(30 \mathrm{mg}$ twice daily) doses of osilodrostat.

US Food and Drug Administration guidance on DDIs classifies the inhibitory effect of an investigational compound according to the following criteria: strong inhibitor, $\geq 5$-fold increase in AUC; moderate inhibitor, $\geq 2$ - but $<5$ fold increase in AUC; weak inhibitor, $\geq 1.25$ - but $<2$-fold increase in AUC [22]. Based on these criteria, the current study indicates that a single dose of $50 \mathrm{mg}$ osilodrostat is a weak inhibitor of CYP2D6 and CYP3A4/5 and a moderate inhibitor of CYP1A2 and CYP2C19. Predicted effects after multiple 30-mg twice-daily doses of osilodrostat resulted in the same classification for inhibition of these CYP enzymes.

The finding that osilodrostat has low inhibitory impact on CYP3A4/5 is clinically relevant, particularly given that commonly prescribed medications that may be used to treat Cushing's disease, such as amlodipine and nifedipine (antihypertensive medications), as well as simvastatin (statin), are CYP3A4 substrates. By contrast, ketoconazole, used off-label for several decades to reduce cortisol levels in Cushing's disease and recently approved in Europe for the treatment of endogenous Cushing's syndrome, is a potent inhibitor of CYP3A4 [23]. Pharmacokinetic studies have shown up to a 16-fold increase in midazolam AUC after ketoconazole administration [24-26]. This would classify ketoconazole as a strong inhibitor ( $>5$-fold change) of CYP3A4 based on Food and Drug Administration guidance [22]. As a result, a considerable number of clinically important medications would be contraindicated if ketoconazole is administered [23]. Of the other current medical therapies available to treat Cushing's disease, data on DDI potential are mostly limited to the prescribing information, although mifepristone has been shown to be a strong inhibitor of CYP3A4 [27], while mitotane is a strong inducer [28]. Co-administration of either of these agents with other therapeutics metabolized by CYP3A4 is likely to have an effect on systemic exposure of co-administered medications, potentially causing an unwanted DDI.

\section{Conclusions}

A single dose of $50 \mathrm{mg}$ of osilodrostat showed weak inhibition of CYP3A4/5- and CYP2D6-mediated interactions, and weak-to-moderate inhibition of CYP2C19- and CYP1A2-mediated interactions. Metabolic drug interactions at lower therapeutic doses of osilodrostat $(<30 \mathrm{mg}$ twice daily, which corresponds to the single-dose exposure at $50 \mathrm{mg}$ in this study) with medications taken concurrently to treat common co-morbidities in patients with Cushing's disease are expected to be even weaker. This provides further support for osilodrostat as a future treatment for Cushing's disease, particularly in the light of recent treatment guidelines for Cushing's disease [1] that recommend clinicians be cautious and check for potential DDIs before starting or adding agents that treat hypercortisolism.

\section{Compliance with Ethical Standards}

Funding This study was funded by Novartis Pharma AG. Financial support for medical editorial assistance was provided by Novartis Pharmaceuticals Corporation. Medical editorial assistance was provided by Richard Ogilvy-Stewart, Mudskipper Business Limited, UK.

Conflict of interest SA is an employee of PAREXEL. NS, CD, SK, LW, BZ, HG, DYC, and HJE are employees of Novartis. LT is an employee of Merck \& Co. and a former employee of Novartis and owned Novartis stock. APT is an employee of PAREXEL and a former employee of Novartis.

Ethics Approval All procedures performed in studies involving human participants were approved by an independent ethics committee and conducted in accordance with the 1964 Declaration of Helsinki and its later amendments or comparable ethical standards.

Informed Consent Informed consent was obtained from all individual participants included in the study.

Open Access This article is distributed under the terms of the Creative Commons Attribution-NonCommercial 4.0 International 
License (http://creativecommons.org/licenses/by-nc/4.0/), which permits any noncommercial use, distribution, and reproduction in any medium, provided you give appropriate credit to the original author(s) and the source, provide a link to the Creative Commons license, and indicate if changes were made.

\section{References}

1. Nieman LK, Biller BM, Findling JW, et al. Treatment of Cushing's syndrome: an Endocrine Society clinical practice guideline. J Clin Endocrinol Metab. 2015;100:2807-31.

2. Fleseriu M, Biller BM, Findling JW, et al. Mifepristone, a glucocorticoid receptor antagonist, produces clinical and metabolic benefits in patients with Cushing's syndrome. J Clin Endocrinol Metab. 2012;97:2039-49.

3. Castinetti F, Guignat L, Giraud P, et al. Ketoconazole in Cushing's disease: is it worth a try? J Clin Endocrinol Metab. 2014;99:1623-30.

4. Daniel E, Aylwin S, Mustafa O, et al. Effectiveness of metyrapone in 195 patients with Cushing's syndrome. Endocrine Abstracts. 2014;34:OC6.5.

5. Menard J, Watson C, Rebello S, et al. Hormonal and electrolyte responses to the aldosterone synthase inhibitor LCI699 in sodium depleted healthy subjects. J Am Coll Cardiol. 2010;55(A61):E583.

6. Amar L, Azizi M, Menard J, et al. Aldosterone synthase inhibition with LCI699: a proof-of-concept study in patients with primary aldosteronism. Hypertension. 2010;56:831-8.

7. Calhoun DA, White WB, Krum H, et al. Effects of a novel aldosterone synthase inhibitor for treatment of primary hypertension: results of a randomized, double-blind, placebo- and active-controlled phase 2 trial. Circulation. 2011;124:1945-55.

8. Bertagna X, Pivonello R, Fleseriu M, et al. LCI699, a potent 11bhydroxylase inhibitor, normalizes urinary cortisol in patients with Cushing's disease: results from a multicenter, proof-of-concept study. J Clin Endocrinol Metab. 2014;99:1375-83.

9. Fleseriu M, Pivonello R, Young J, et al. Osilodrostat, a potent oral 11b-hydroxylase inhibitor: 22-week, prospective, phase II study in Cushing's disease. Pituitary. 2016;19:138-48.

10. Li W, Luo S, Rebello S, et al. A semi-automated LC-MS/MS method for the determination of LCI699, a steroid 11 $\beta$-hydroxylase inhibitor, in human plasma. J Chromatogr B Analyt Technol Biomed Life Sci. 2014;960:182-93.

11. Tomaszewski P, Kubiak-Tomaszewska G, Pachecka J. Cytochrome P450 polymorphism: molecular, metabolic, and pharmacogenetic aspects. II. Participation of CYP isoenzymes in the metabolism of endogenous substances and drugs. Acta Pol Pharm. 2008;65:307-18.

12. Zanger UM, Turpeinen M, Klein K, Schwab M. Functional pharmacogenetics/genomics of human cytochromes P450 involved in drug biotransformation. Anal Bioanal Chem. 2008;392:1093-108.
13. Watkins PB. Noninvasive tests of CYP3A enzymes. Pharmacogenetics. 1994;4:171-84.

14. Gibson GG, Plant NJ, Swales KE, et al. Receptor-dependent transcriptional activation of cytochrome P4503A genes: induction mechanisms, species differences and interindividual variation in man. Xenobiotica. 2002;32:165-206.

15. Cholerton S, Daly AK, Idle JR. The role of individual human cytochromes $\mathrm{P} 450$ in drug metabolism and clinical response. Trends Pharmacol Sci. 1992;13:434-9.

16. European Medicines Agency. Guideline on the investigation of drug interactions. 2016. http://www.ema.europa.eu/docs/en_GB/ document_library/Scientific_guideline/2012/07/WC500129606. pdf. Accessed 10 Jan 2017.

17. Huang SM, Strong JM, Zhang L, et al. New era in drug interaction evaluation: US Food and Drug Administration update on CYP enzymes, transporters, and the guidance process. J Clin Pharmacol. 2008;48:662-70.

18. Streetman DS, Bertino JS Jr, Nafziger AN. Phenotyping of drugmetabolizing enzymes in adults: a review of in-vivo cytochrome P450 phenotyping probes. Pharmacogenetics. 2000;10:187-216.

19. Jamei M. Recent advances in development and application of physiologically-based pharmacokinetic (PBPK) models: a transition from academic curiosity to regulatory acceptance. Curr Pharmacol Rep. 2016;2:161-9.

20. Sinha V, Zhao P, Huang SM, Zineh I. Physiologically based pharmacokinetic modeling: from regulatory science to regulatory policy. Clin Pharmacol Ther. 2014;95:478-80.

21. Zhao P, Zhang L, Grillo JA, et al. Applications of physiologically based pharmacokinetic (PBPK) modeling and simulation during regulatory review. Clin Pharmacol Ther. 2011;89:259-67.

22. FDA. Guidance for industry. Drug interaction studies: study design, data analysis, implications for dosing, and labeling recommendations. 2012. http://www.fda.gov/downloads/drugs/guidancecompliance regulatoryinformation/guidances/ucm292362.pdf. Accessed 10 Jan 2017.

23. HRA Pharma. Ketoconazole HRA summary of product characteristics. 2015. http://www.medicines.org.uk/emc/medicine/ 30077. Accessed 10 Jan 2017.

24. Lam YW, Alfaro CL, Ereshefsky L, Miller M. Pharmacokinetic and pharmacodynamic interactions of oral midazolam with ketoconazole, fluoxetine, fluvoxamine, and nefazodone. J Clin Pharmacol. 2003;43:1274-82.

25. Lee JI, Chaves-Gnecco D, Amico JA, et al. Application of semisimultaneous midazolam administration for hepatic and intestinal cytochrome P450 3A phenotyping. Clin Pharmacol Ther. 2002;72:718-28.

26. Olkkola KT, Backman JT, Neuvonen PJ. Midazolam should be avoided in patients receiving the systemic antimycotics ketoconazole or itraconazole. Clin Pharmacol Ther. 1994;55:481-5.

27. He K, Woolf TF, Hollenberg PF. Mechanism-based inactivation of cytochrome P-450-3A4 by mifepristone (RU486). J Pharmacol Exp Ther. 1999;288:791-7.

28. van Erp NP, Guchelaar HJ, Ploeger BA, et al. Mitotane has a strong and a durable inducing effect on CYP3A4 activity. Eur J Endocrinol. 2011;164:621-6. 